\title{
COMPACT SEMIGROUPS OF POSITIVE MATRICES
}

\author{
T. T. West
}

School of Mathematics, Trinity College Dublin

\section{$\$ 1 \quad$ Introduction}

The spectral theory of compact monothetic semigroups of linear operators examined by Kaashoek and West in [1], [2] together with two block matrix theorems where the blocks are either strictly positive or zero are used to give an exposition of Perron-Frobenius theory of positive matrices. The approach in this paper is based on ideas of Smyth and West developed in [4], [5].

We consider a linear operator $\mathrm{T}$ in finite dimensions which has a matrix representation [T] relative to a given basis. Where there is no ambiguity we often write the matrix as $T$. $T \geq 0$ if $[\mathrm{T}]_{\mathrm{ij}} \geq 0(\forall \mathrm{i}, \mathrm{j})$ while $\mathrm{T}>0$ if $[\mathrm{T}]_{\mathrm{ij}}>0(\forall \mathrm{i}, \mathrm{j})$. The spectrum and spectral radius of $\mathrm{T}$ will be denoted by $\sigma(\mathrm{T})$ and $\mathrm{r}(\mathrm{T})$ respectively. The trace of $\mathrm{T}$ (the sum of its eigenvalues) will be written as $\operatorname{tr}(\mathrm{T})$, and the peripheral spectrum will be denoted by $\pi(\mathrm{T})=\{\lambda \in \sigma(\mathrm{T}) ;|\lambda|=r(\mathrm{~T})\}$. The $i^{\text {th }}$ row and $j^{\text {th }}$ column of $T$ relative to the given basis will be written $\operatorname{row}_{i}(T)$ and $\operatorname{col}_{j}(T)$ and the diagonal of $\mathrm{T}$ will be denoted $\operatorname{diag}(\mathrm{T})$. The spectral projection of $\mathrm{T}$ relative to $\pi(\mathrm{T})$ will be written $\mathrm{P}_{\pi}$.

Smyth [5] has introduced a hierarchy of subsets of matrices $\mathrm{T} \geq 0$.

Definitions. (i) $\quad \mathrm{T}$ is positive if $\mathrm{T}>0$;

(ii) $\quad \mathrm{T}$ is primitive if $\mathrm{T}^{\mathrm{k}}>0$ for some positive integer $\mathrm{k}$;

(iii) $\quad \mathrm{T}$ is connected if $\forall \mathrm{i}, \mathrm{j} \exists$ a positive integer $\mathrm{k}$ such that $\left[\mathrm{T}^{\mathrm{k}}\right]_{\mathrm{ij}}>0$;

(iv) $\quad \mathrm{T}$ is potent if $\operatorname{diag}\left(\mathrm{T}^{\mathrm{k}}\right)>0$ for some positive integer $\mathrm{k}$;

(v) T is zero-free if no row or column is zero;

(vi) $\mathrm{T}$ has positive spectral radius. 


\section{Remarks.}

The above sets are strictly ordered by inclusion. $\mathrm{T}$ is connected if and only if there exists a positive integer $\mathrm{p}$ such that $\mathrm{T}+\mathrm{T}^{2}+\ldots . .+\mathrm{T}^{\mathrm{p}}>0$. It is also connected if no basis permutation results in a block representation

$$
T=\left(\begin{array}{ll}
U & 0 \\
V & W
\end{array}\right)
$$

where $\mathrm{U}$ and $\mathrm{W}$ are square blocks. If $\mathrm{S}$ and $\mathrm{T}$ are zero-free then so is ST. It follows that if $\mathrm{T}$ is zero-free $\mathrm{r}(\mathrm{T})>0$. Note also that all these subsets are invariant under a basis permutation, and that if $\mathrm{S} \geq \mathrm{T}$ and $\mathrm{T}$ is contained in any one of these sets then so is $\mathrm{S}$.

The following upgrading lemma will be important.

Lemma 1. If $S, T \geq 0, T \neq 0$ and $S$ is connected then $S T=T S$ implies $T$ is potent.

Proof. Observe that by replacing $S$ by $S+S^{2}+\ldots \ldots+S^{p}$ for sufficiently large $p$ we may assume that $S>0$. First we show that under these conditions $T$ is zero-free. As $T \neq 0,[T]_{i j}>0$ for some $\mathrm{i}, \mathrm{j}$. Then $[\mathrm{STT}]_{\mathrm{kj}} \geq[\mathrm{S}]_{\mathrm{ki}}[\mathrm{T}]_{\mathrm{ij}}>0(\forall \mathrm{k})$, therefore $[\mathrm{TS}]_{\mathrm{kj}}>0(\forall \mathrm{k})$ so $\operatorname{row}_{\mathrm{k}}(\mathrm{T})$ is nonzero $(\forall \mathrm{k})$ and taking transposes gives the same result for columns.

We prove that $\mathrm{T}$ is potent by induction on the size of the matrix. The result is trivially true for $1 \mathrm{x} 1$ matrices so assume that it holds for $\mathrm{k} \times \mathrm{k}$ matrices $(\mathrm{k}=1, \ldots . ., \mathrm{n}-1)$.

If $\mathrm{T}(\mathrm{n} \times \mathrm{n})$ is connected the result is trivially true so assume that $\mathrm{T}$ is not connected. Then by a basis permutation $\mathrm{T}$ has lower triangular block form

$$
T=\left(\begin{array}{cc}
T_{11} & 0 \\
T_{21} & T_{22}
\end{array}\right)
$$

where $\mathrm{T}_{11}$ and $\mathrm{T}_{22}$ are square blocks which must be non-zero as $\mathrm{T}$ is zero-free. Corresponding to this decomposition

$$
S=\left(\begin{array}{ll}
S_{11} & S_{12} \\
S_{21} & S_{22}
\end{array}\right) \text { with } S_{\mathrm{ij}}>0(\forall \mathrm{i}, \mathrm{j}) .
$$

Since $S$ and $T$ commute we have $T_{11} S_{11}=S_{11} T_{11}+S_{12} T_{21}$. But $\operatorname{tr}\left(T_{11} S_{11}\right)=\operatorname{tr}\left(S_{11} T_{11}\right)$ so $\operatorname{tr}\left(S_{12} T_{21}\right)=0$. By positivity $T_{21}=0$ but now $T_{11}$ commutes with $S_{11}>0$ and $T_{22}$ commutes with $\mathrm{S}_{22}>0$. By our induction hypothesis, these blocks are both potent hence so is $\mathrm{T}$.

\section{\$2 Compact Semigroups}


Let $\mathrm{T}$ be connected. Then $\mathrm{r}(\mathrm{T})>0$ so, without loss of generality, we take $\mathrm{r}(\mathrm{T})=1$.

Proposition 2. If $T$ is connected and $r(T)=1$ then $\left\|T^{n}\right\| \leq M(n=1,2, \ldots \ldots)$.

Proof. $S(T)=\operatorname{cl}\left\{T^{n} ; n \geq 1\right\}$ is a closed monothetic (singly generated) semigroup, further $W=R^{+} S(T)$ is also a semigroup and $W_{1}=\{W \in W:\|W\|=1\}$ is a closed, bounded, nonempty subset of $W$ which is therefore compact. If $W \in W_{1}$ then $W$ is potent by Lemma 1 , hence $\mathrm{r}(\mathrm{W})>0$ for each $\mathrm{W} \in \mathrm{W}_{1}$. Further the spectral radius is norm-continuous and therefore attains its minimum $\mu$ on the compact set $W_{1}$. Then

$$
\mathrm{r}(\mathrm{W}) \geq \mu>0 \quad\left(\mathrm{~W} \in \mathrm{W}_{1}\right) \text { and so } \mathrm{r}(\mathrm{S})\|\mathrm{S}\|^{-1} \geq \mu(\mathrm{S} \in \mathrm{S}(\mathrm{T})) \text {. }
$$

But $r(S)=1$ for $S \in S(T)$ hence $\|S\| \leq \mu^{-1} \quad(S \in S(T))$ and the monothetic semigroup $S(T)$ is closed and bounded, therefore compact.

The structure theory for such compact monothetic semigroups [1], [2] now shows that $S$ (T) contains a unique idempotent which is $\mathrm{P}_{\pi}$, that all eigenvalues in $\pi(\mathrm{T})$ are simple and that

$$
G(T)=P_{\pi} S(T)=S\left(P_{\pi} T\right)
$$

is a compact monothetic group with unit $\mathrm{P}_{\pi}$ consisting of all limit points of $S(\mathrm{~T})$. Note that $\mathrm{P}_{\pi} \geq 0$ and that, since $\mathrm{P}_{\pi}$ commutes with $\mathrm{T}, \mathrm{P}_{\pi}$ is potent hence $\operatorname{diag}\left(\mathrm{P}_{\pi}\right)>0$.

Further if $\pi(\mathrm{T})=\left\{\lambda_{1}, \lambda_{2}, \ldots, \lambda_{\mathrm{k}}\right\}$ then $G(\mathrm{~T})$ is isomorphic to the compact monothetic subgroup of $C^{\mathrm{k}} \mathrm{cl}\left\{\kappa_{1}^{\mathrm{n}}, \kappa_{2}^{\mathrm{n}}, \ldots \ldots, \kappa_{\mathrm{k}}^{\mathrm{n}} ; \mathrm{n} \mu 1\right\}$. By hypothesis $\mathrm{T}$ is potent hence $\operatorname{diag}\left(\mathrm{T}^{\mathrm{p}}\right)>0$ for some positive integer p. It follows now by [4], Proposition 2 that $\pi\left(\mathrm{T}^{\mathrm{p}}\right)=\{1\}$ hence the peripheral eigenvalues of $\mathrm{T}$ are all $\mathrm{p}^{\text {th }}$ roots of unity. It follows at once by the above isomorphism for $G(\mathrm{~T})$ that $G(\mathrm{~T})$ is a finite cyclic group generated by $\mathrm{T}$. Put $\mathrm{R}=\mathrm{P}_{\pi} \mathrm{T}$. Obviously $\mathrm{R} \geq 0$ and, since $\mathrm{T}$ is connected, $T_{p}=\sum_{n=1}^{p} T^{n}>0$ for sufficiently large $\mathrm{p}$. Further $R_{p}=\sum_{n=1}^{p} R^{n}=P T_{p} . \quad$ Now since $\operatorname{diag}(\mathrm{P})>0, \quad\left[\mathrm{R}_{\mathrm{p}}\right]_{\mathrm{ij}}=\left[\mathrm{PT}_{\mathrm{p}}\right]_{\mathrm{ij}} \geq[\mathrm{P}]_{\mathrm{ii}}\left[\mathrm{T}_{\mathrm{p}}\right]_{\mathrm{ij}}>0 \quad(\forall \mathrm{i}, \mathrm{j})$ so $R_{p}>0$ and $R$ is connected. 
Consider the simple case in which $\pi(T)=\{1\}$. Then by the isomorphism $G(T)$ consists of one element $\mathrm{P}_{\pi}$ so $\mathrm{T}^{\mathrm{n}} \rightarrow \mathrm{P}(\mathrm{n} \rightarrow \infty)$. Thus for a general connected $\mathrm{T}$ as we have seen $\pi\left(\mathrm{T}^{\mathrm{p}}\right)=$ $\{1\}$ for a positive integer $\mathrm{p}$ so $G(T)$ is a finite cyclic group and $\left(T^{\mathrm{p}}\right)^{\mathrm{n}}=\mathrm{T}^{\mathrm{pn}} \rightarrow \mathrm{P}_{\pi} \quad(\mathrm{n} \rightarrow \infty)$.

We now use these results to characterise primitive matrices. Note that if $T^{k}>0$ then $T^{k+1}=$ $\mathrm{T}^{\mathrm{k}} \mathrm{T}$ is the product of a positive with a zero-free matrix which is therefore positive. Hence $\mathrm{T}^{\mathrm{k}+\mathrm{n}}>0$ for all positive integers $\mathrm{n}$.

Proposition 3. Let $T \geq 0$ with $r(T)=1$. Then the following are equivalent :

(i) $T$ is primitive ;

(ii) $T$ is connected and $\pi(T)=\{1\}$;

(iii) $T$ is connected and $T^{n} \rightarrow P_{\pi}$;

(iv) $T$ is connected and $G(\mathrm{~T})=\left\{P_{\pi}\right\}$.

Proof. (i) $\Leftrightarrow$ (ii). Assume that $\mathrm{T}$ is primitive. Then $\mathrm{T}$ is connected. Now $\mathrm{T}^{\mathrm{k}}>0$ for some $\mathrm{k}$ hence $\operatorname{diag}\left(\mathrm{T}^{\mathrm{k}}\right)>0$ therefore $\mathrm{T}^{\mathrm{kn}} \rightarrow \mathrm{P}_{\pi}(\mathrm{n} \rightarrow \infty)$ so $\mathrm{T}^{\mathrm{k}} \mathrm{P}_{\pi}=\mathrm{P}_{\pi}$.

Next we show that $P_{\pi}>0$. Suppose not, then $\left[P_{\pi}\right]_{i j}=0$ for some $i, j$ hence $\left[\mathrm{T}^{\mathrm{k}} \mathrm{P}_{\mathrm{o}}\right]_{\mathrm{ij}}=\sum_{\mathrm{m}=1}^{\mathrm{n}}\left[\mathrm{T}^{\mathrm{k}}\right]_{\mathrm{im}}\left[\mathrm{P}_{\mathrm{o}}\right]_{\mathrm{mj}}=0$, thus, using positivity, $\left[\mathrm{P}_{\pi}\right]_{\mathrm{mj}}=0(\forall \mathrm{m})$, that is $\operatorname{col}_{\mathrm{j}}\left(\mathrm{P}_{\pi}\right)=0$ contradicting the fact that $\operatorname{diag}\left(\mathrm{P}_{\pi}\right)>0$. Hence $\mathrm{P}_{\pi}>0$ hence $\operatorname{rank}\left(\mathrm{P}_{\pi}\right)=1$ therefore $\pi(\mathrm{T})=\{1\}$. Conversely let $\mathrm{T}$ be connected with $\pi(\mathrm{T})=\{1\}$. Then $\mathrm{T}^{\mathrm{n}} \rightarrow \mathrm{P}_{\pi}(\mathrm{n} \rightarrow \infty)$ so $\mathrm{T}^{\mathrm{n}} \mathrm{P}_{\pi}=\mathrm{P}_{\pi} \mathrm{T}^{\mathrm{n}}=\mathrm{P}_{\pi}$ $(\forall n)$. Suppose $\left[\mathrm{P}_{\pi}\right]_{\mathrm{ij}}=0$ for some $\mathrm{i}, \mathrm{j}$. Since $\mathrm{T}$ is connected $\left[\mathrm{T}^{\mathrm{k}}\right]_{\mathrm{ij}}>0$ for some $\mathrm{k}$, therefore $0=\left[\mathrm{P}_{\pi}\right]_{\mathrm{ij}} \geq\left[\mathrm{T}^{\mathrm{k}}\right]_{\mathrm{ij}}\left[\mathrm{P}_{\pi}\right]_{\mathrm{jj}}$ so $\left[\mathrm{P}_{\pi}\right]_{\mathrm{jj}}=0$ which contradicts the fact that $\operatorname{diag}\left(\mathrm{P}_{\pi}\right)>0$. Hence $\mathrm{P}_{\pi}>0$ and because $T^{n} \rightarrow P_{\pi}(n \rightarrow \infty)$ it now follows that $T^{m}>0$ for some $m$.

(ii) $\Leftrightarrow$ (iii). The preceding remarks show that if $\pi(\mathrm{T})=\{1\}$ then $\mathrm{T}^{\mathrm{n}} \rightarrow \mathrm{P}_{\pi}$. Conversely if $\mathrm{T}^{\mathrm{n}} \rightarrow \mathrm{P}_{\pi}$ then $\pi\left(\mathrm{T}^{\mathrm{n}}\right) \rightarrow \pi\left(\mathrm{P}_{\pi}\right)=\{1\}(\mathrm{n} \rightarrow \infty)$ which implies $\pi(\mathrm{T})=\{1\}$.

(ii) $\Leftrightarrow$ (iv). It is clear from our remarks prior to Proposition 3 that, if $\mathrm{T}$ is connected and $\mathrm{r}(\mathrm{T})=1$, then $\pi(\mathrm{T})=\{1\} \Leftrightarrow G(\mathrm{~T})=\left\{\mathrm{P}_{\pi}\right\}$. 


\section{$\S 3$ Block Matrix Representations}

The following block representation of a zero-free idempotent $\mathrm{P} \geq 0$ is well known ([3], Lemma 5.1.9).

Proposition 4. Let $P \geq 0$ be a zero-free idempotent matrix of rank $h$ then via a basis permutation $P$ has the block matrix representation

$$
\mathrm{P}=\left(\begin{array}{cccc}
\mathrm{P}_{11} & 0 & . . & 0 \\
0 & \mathrm{P}_{22} & . . & 0 \\
. . & . . & . . & . . \\
0 & 0 & . . & \mathrm{P}_{h h}
\end{array}\right)
$$

where all the off diagonal blocks are zero, the diagonal blocks are square and $P_{i i}>0$ is an idempotent of rank $1(i=1, \ldots \ldots, h)$.

Proof. If $\mathrm{P}$ is connected then $\mathrm{P}>0, \mathrm{~h}=1$ and the result holds. Assume then that via a basis rearrangement $\mathrm{P}$ has the block representation $P=\left(\begin{array}{cc}U & 0 \\ V & W\end{array}\right)$ As $\mathrm{P}^{2}=\mathrm{P}>0$ hence $\mathrm{U}^{2}=\mathrm{U} \geq 0$, and $\mathrm{W}^{2}=\mathrm{W} \geq 0$ and $\mathrm{VU}+\mathrm{WV}=\mathrm{V}$ thus $\mathrm{WVU}+\mathrm{WV}=\mathrm{WV}$, so $\mathrm{WVU}=0$. Now as $\mathrm{P}$ is zero-free $\mathrm{U}$ has no zero rows and $\mathrm{W}$ has no zero columns then $\mathrm{V}=0$. Then $\mathrm{U}$ and $\mathrm{W}$ are both idempotents $\geq 0 . \quad P=\left(\begin{array}{cc}U & 0 \\ 0 & W\end{array}\right)$ is zero-free hence so are $\mathrm{U}$ and $\mathrm{W}$. The result follows by further reduction until the diagonal blocks are all connected idempotents and therefore $>0$.

(For a general block representation of $\geq 0$ idempotent matrices see [3], Lemma 5.1.9)

Now let $T \geq 0$ be connected with $r(T)=1$. If $P_{\pi} T=R$ then $G(T)=S(R)$ is a finite cyclic group and we can find $\mathrm{S} \in \mathrm{S}(\mathrm{R})$ such that $\mathrm{P}_{\pi} \mathrm{R}=\mathrm{RP}_{\pi}=\mathrm{R}, \mathrm{P}_{\pi} \mathrm{S}=\mathrm{SP}_{\pi}=\mathrm{S}, \mathrm{SR}=\mathrm{P}_{\pi}=\mathrm{RS}$. Further $\mathrm{R}, \mathrm{S} \geq 0$ and $\mathrm{R}$ is connected, also $\mathrm{R}, \mathrm{S}$ are zero-free since $\mathrm{P}_{\pi}$ is, so each block row of the block matrix R (and S) corresponding to the block representation of $\mathrm{P}$ will have at least one non-zero block. 
Suppose that $R_{i j}$ is a block which is not zero. Then we have $s, t$ such that $\left[R_{i j}\right]_{s t}>0$. Now $\mathrm{R}=\mathrm{PRP}$ and the blocks $\mathrm{P}_{\mathrm{ii}}, \mathrm{P}_{\mathrm{jj}}>0(\forall \mathrm{i}, \mathrm{j})$ so, for every compatible pair $\mathrm{m}, \mathrm{n}$, $\left[\mathrm{R}_{\mathrm{ij}}\right]_{\mathrm{mn}} \geq\left[\mathrm{P}_{\mathrm{ii}}\right]_{\mathrm{ms}}\left[\mathrm{R}_{\mathrm{ij}}\right]_{\mathrm{st}}\left[\mathrm{P}_{\mathrm{jj}}\right]_{\mathrm{tn}}>0$, thus the block $\mathrm{R}_{\mathrm{ij}}>0$.

We now generalise a well known result for $\geq 0$ invertible matrices.

Proposition 5. Let $R, S, P$ be as above and such that $R S=P=S R$. Then $R$ (and $S$ ) have exactly one block in each row or column which is $>0$ and the remaining blocks are zero.

Proof. Since $\mathrm{P}$ is zero-free so are $\mathrm{R}$ and $\mathrm{S}$ so they both have (at least) one non-zero block in each row or column. Suppose $\mathrm{R}_{1 \mathrm{k}}>0$, then $\mathrm{RS}=\mathrm{P}$ so the block $P_{1 i}=(R S)_{1 i}=\sum_{j=1}^{h} R_{1 j} S_{j i}=0$ $(\forall \mathrm{i}>1)$. Taking $\mathrm{j}=\mathrm{k}$ gives $\mathrm{S}_{\mathrm{ki}}=0(\forall \mathrm{i}>1)$, by positivity, that is $\operatorname{blockrow}_{\mathrm{k}}(\mathrm{S})$ has exactly one non-zero block $S_{\mathrm{kl}}$ and $S_{\mathrm{kl}}>0$. Reversing the order and taking transposes gives the required result.

Replacing each positive block of $\mathrm{R}$ with the number one and each zero block with the number zero gives an $\mathrm{h} x \mathrm{~h}$ permutation matrix, which, since $\mathrm{R}$ is connected must be a single cycle. A basis permutation then ensures that $\mathrm{R}$ has an $\mathrm{h} \times \mathrm{h}$ block representation of the form

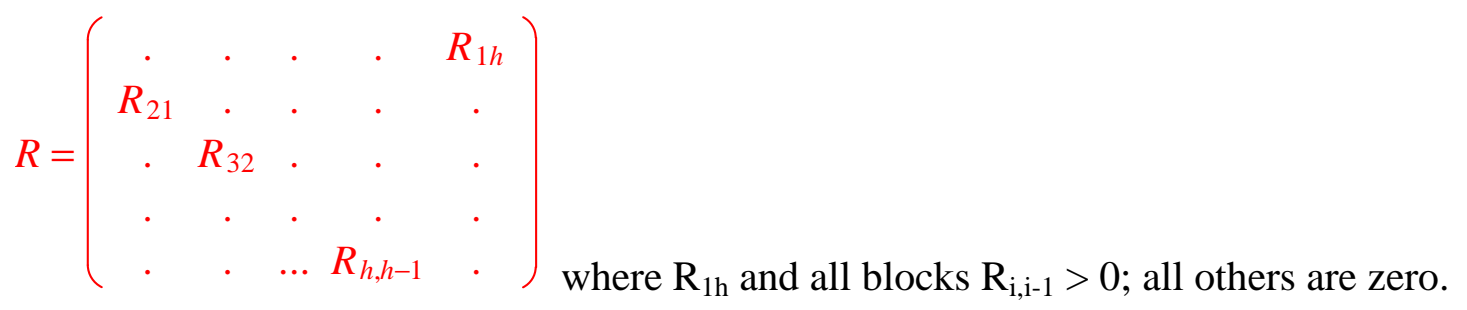

Consider the equivalent $h \times h$ block representation $T$. For each $i, j$ block $R_{i j}=(T P)_{i j} \geq T_{i j} P_{j j}$. Since $P_{j j}>0$ we deduce that $R_{i j}=0$ implies that $T_{i j}=0$. Thus the block representation $T$ is subservient to that of $\mathrm{R}$, in the sense that its non-zero blocks can only occur in positions $\mathrm{i}, \mathrm{j}$ in which $R_{i j}>0$. 
Finally observe that by [1], [2] if $|\lambda|=1$ and $\mathrm{P}(\lambda ; \mathrm{R})$ denotes the spectral projection of the point $\lambda$ associated with the linear operator $\mathrm{R}$ that $\mathrm{n}^{-1} \sum_{\mathrm{k}=1}^{\mathrm{n}} \kappa^{-\mathrm{k}} \mathrm{R}^{\mathrm{k}} \delta \mathrm{P}(\kappa ; \mathrm{R})(\mathrm{n} \delta \equiv)$ where $\mathrm{P}(\lambda ; \mathrm{R}) \neq 0$ if and only if $\lambda \in \pi(\mathrm{R})$. But since $\mathrm{R}^{\mathrm{h}+1}=\mathrm{R}$ choosing $\lambda$ such that $\lambda^{\mathrm{h}}=1$ gives $h^{-1} \sum_{k=1}^{h} \kappa^{-k} R^{k}=P(\kappa ; R)$. To show that every $h^{\text {th }}$ root of unity is an eigenvalue of $R$ observe that, from our $\mathrm{h} x \mathrm{~h}$ block representation of $\mathrm{R}, \operatorname{diag}\left(\mathrm{R}^{\mathrm{k}}\right)=0 \quad(\mathrm{k}=1, \ldots . ., \mathrm{h}-1)$; but that $\operatorname{diag}\left(\mathrm{R}^{\mathrm{h}}\right)=\operatorname{diag}(\mathrm{P})>0$. Thus $\mathrm{P}(\lambda ; \mathrm{R}) \neq 0$ if, and only if, $\lambda^{\mathrm{h}}=1$.

With this block matrix representation for $\mathrm{T}$ let $\mathrm{D}$ be the block diagonal matrix

$$
\mathrm{D}=\operatorname{diag}\left(\mathrm{e}^{\mathrm{i} \omega} \mathrm{I}_{1}, \mathrm{e}^{2 \mathrm{i} \omega} \mathrm{I}_{2}, \ldots \ldots \ldots, \mathrm{e}^{\mathrm{hi} \omega} \mathrm{I}_{\mathrm{h}}\right)
$$

where $\omega=2 \pi / h$. Then $D_{T D}^{-1}=e^{i \omega} T$ and the whole spectral theory of $T$ is invariant under rotations by multiples of $\omega$.

Now recall that the trace of $\mathrm{T}^{\mathrm{n}}$ is given by $\operatorname{tr}\left(\mathrm{T}^{\mathrm{n}}\right)=\sum_{\mathrm{i}=1}^{\mathrm{p}} \kappa_{\mathrm{i}}^{\mathrm{n}}$ where $\sigma(T)=\left\{\lambda_{\mathrm{i}} ; 1 \leq \mathrm{i} \leq \mathrm{p}\right\}$.

Proposition 6. If $T \geq 0$ and $r(T)=1$ then $T$ is primitive $\Leftrightarrow \quad T$ is connected and $\operatorname{tr}\left(T^{n}\right) \rightarrow 1 \quad(n \rightarrow \infty)$.

Proof. Let $T$ be primitive. Then by Proposition 3(iii) $T^{n} \rightarrow P_{\pi}$ so $\operatorname{tr}\left(T^{n}\right) \rightarrow \operatorname{tr}\left(P_{\pi}\right)=1$.

Conversely let $\mathrm{T}$ be connected and, as before, set $\mathrm{P}_{\pi} \mathrm{T}=\mathrm{R}$. The above discussion shows that the eigenvalues of $R$ are precisely the $h^{\text {th }}$ roots of unity for some positive integer $h$ and therefore $\operatorname{tr}\left(R^{n}\right)=h$ whenever $n$ is divisible by $h$, otherwise $\operatorname{tr}\left(R^{n}\right)=0$. However $\pi(T)=\pi(R)$ and as $n \rightarrow \infty$ the $n^{\text {th }}$ powers of $\sigma(T) \backslash \pi(T)$ go to zero. Hence $\operatorname{tr}\left(T^{n}\right)-\operatorname{tr}\left(R^{n}\right) \rightarrow 0$ as $n \rightarrow \infty$ so $\operatorname{tr}\left(\mathrm{T}^{\mathrm{n}}\right)$ is convergent if and only if $\mathrm{h}=1$ and the limit in this particular case is always 1

Corollary. If $T \geq 0$ and $r(T)=1$ then $T$ is primitive $\Leftrightarrow \quad T$ is connected and $\left\{\operatorname{tr}\left(T^{n}\right)\right\}_{1}^{\infty}$ is a convergent sequence. 


\section{REFERENCES}

1. M.A.Kaashoek and T.T.West, Locally compact monothetic semialgebras, Proc. London Math. Soc. 18(1968), 428-438.

2. M.A.Kaashoek and T.T.West, Locally Compact Semi-Algebras with applications to Spectral Theory of Positive Operators, Amsterdam, 1974.

3. H.Radjavi and P.Rosenthal, Simultaneous Triangularisation, New York, 2000.

4. M.R.F.Smyth and T.T.West, The minimum diagonal element of a positive matrix, Studia Math 131(1) 1998, 95-99.

5. M.R.F.Smyth, A spectral theoretic proof of Perron-Frobenius, Proc. Roy. Irish Acad. (to appear). 